\title{
Interactive Climbing Route Design Using a Simulated Virtual Climber
}

\author{
Jonas Pfeil $1^{1,2}$ \\ Jun Mitani ${ }^{1,3}$ \\ Takeo Igarashi ${ }^{1,4}$
}

${ }^{1}$ JST ERATO Igarashi Design Interface Project, Tokyo, Japan

${ }^{2} \mathrm{TU}$ Berlin ${ }^{3}$ University of Tsukuba ${ }^{4}$ The University of Tokyo

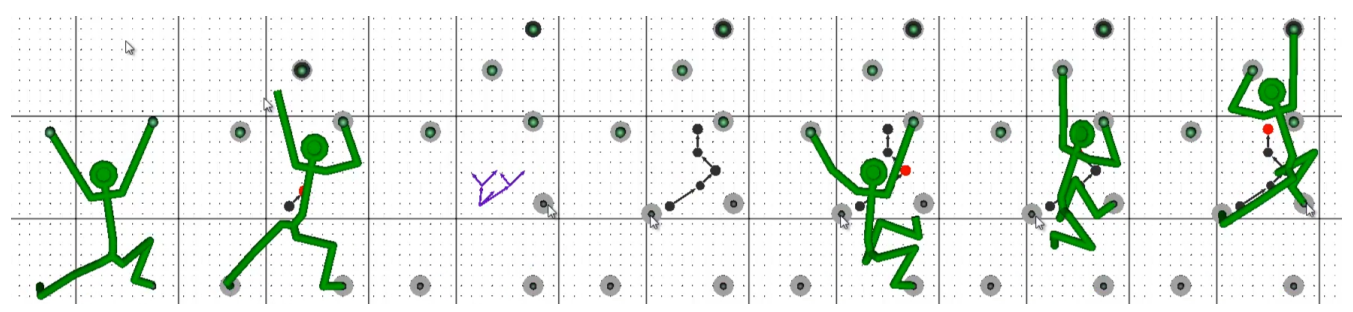

Figure 1: Usage of the climbing route designer (left to right): Start configuration; User added an easily reachable hold; User added more holds - search is running; Route is found and visualized; Climbing animation is shown.

\section{Introduction}

Route setting is the process of creating climbing routes for the sport of free climbing. By fastening climbing holds to artificial climbing walls, a route emerges that provides a challenge for climbers. It is a difficult process usually requiring either a very skilled climber or a lot of time and test climbs. We propose a climbing route designer that aims to enable even novice climbers to create interesting and challenging routes by designing them on the computer using a simulated climber to analyze the route and visualize the result.

While the user places climbing holds on the wall, the software tries to find a route from the start configuration of the virtual climber to a finish hold (see Figure 1). Each hand or foot is moved individually and the static poses are tested for feasibility and scored for difficulty. The progress of the search is visualized to give the user immediate feedback. When finished, the climber is animated and the resulting route is shown, including the difficulty of the individual poses. In our current implementation static poses are calculated by a heuristic, not using physics based calculation. Dynamics are not considered and movement between the poses is simply interpolated. Using this prototype of the virtual climber it was possible to successfully test the user interface concept and collect a lot of feedback and ideas. We conducted an informal user study with climbers that had varying degrees of route setting experience.

\section{Previous Work}

Our work is inspired by recent design tools for novices [2009]. These tools run physical simulation in the background to provide guiding information to the user during design. One example is Sensitive Couture [2011] which helps garment design by running drape simulation. Similarly, our system helps the design of climbing routes by simulating climbing behavior in the background.

Ouchi et al. created a model for the climbing behavior of children [2010]. They collected data from children climbing an array of uniform holds with embedded sensors and built a model for the prediction of climbing behavior. Unfortunately in free climbing the route is defined by the selection and placement of thousands of very differently shaped holds which define the movement of the climber. This makes this data-driven approach infeasible for our purpose.

There have been attempts to make robots free climb on climbing walls as opposed to using mechanics designed for a specific surface like suction cups. One such algorithm was developed by Bretl et al. for the LEMUR IIb robot [2006]. It finds transitions between stances, associations of hands and feet to holds. Their approach is very promising but unfortunately still too slow to be used interactively. Kalisiak and Panne proposed a 2D, grasp-based motion planning algorithm for virtual characters using pose heuristics [2001].

Phillips and Bradley created a system that generates chaotic variations of existing routes which are defined by entering free-form text for each hand move [2010]. In contrast, our system lets the user design a route from scratch, defining every move to his liking.

\section{Climbing Route Designer}

Our software lets the user edit a climbing route by placing holds on a flat climbing wall while information that is obtained through the simulation of the virtual climber is visualized. At the beginning of a session, the climber is already located on four pre-placed holds. A path planning algorithm starts as soon as the user begins adding holds to the wall. The search tries to generate movement for the virtual climber so that it reaches the finish hold. It is defined as the hold farthest away from the start configuration of the climber.

User Input: Normal holds can be placed by left-clicking while right-clicking places a foot hold. Normal holds work for both hand and feet while foot holds are for feet only. Clicking a hold again deletes it. Holds can be dragged around. The user can switch to a spray-paint mode which places normal and foot holds in a random pattern while the user clicks and drags. There is also a button that removes holds unused in the found climbing route.

Virtual Climber: Our virtual climber implementation is a prototype providing rough estimates of climbing poses and movement but runs at interactive speeds. It iteratively searches for a climbing route while the user places, moves and deletes holds. Holds are basically ball joints, we only differentiate between normal holds and foot holds. Poses are calculated at each 4-hold-stance by minimizing a cost function to mimic rest poses of human climbers:

$$
f=w *\left(\text { climber }_{y}-\text { holdMean }_{y}\right)+\sum_{i=1}^{12} \text { bone }_{z}^{i}
$$

where $w=100$ is a weight, climber $_{y}$ is the climbers vertical position, holdMean $y$ is the mean vertical position of the holds in the stance and bone $e_{z}^{i}$ is the distance of bone $i$ from the wall. This 
causes the virtual climber to hang on long arms below the holds and keep his center of mass close to the wall over his feet, both traits of a good climber. We use the SNOPT optimization library [2005] for minimization using additional constraint functions to fix hands and feet to the holds and implement collision with the wall.

The virtual character climbs up the wall moving one hand or foot at a time to a new hold, thus switching to a new stance. We use $\mathrm{A}^{*}$ path finding to determine a sequence of stances from the starting stance to the finish hold. Movement between stances is animated by interpolating the positional constraint of the hand or feet that is being moved and using a different cost function: The sum of the distances of the joints to their interpolated positions. Both pose calculation at stances and the animation between them are cached to avoid unnecessary re-computations while the user edits the route.

The skeleton of the climber has five 3-degrees-of-freedom-joints (DOF) around the hip and torso, two 2-DOF joints at the feet and four 1-DOF-joints at the knees and elbows. Joint constraints are approximated by constraints of the Euler angles used for the rotation.

Visualization: The finish hold is marked with a black circle. While the path finding algorithm is running, the search tree is visualized and holds that have been reached are marked with gray circles (see Figure 2). Once a path is found, only used holds are highlighted. The path is visualized with black arrows and circles at the mean position of the holds in the stance. The circle size reflects the difficulty of the pose (the value of the cost function).
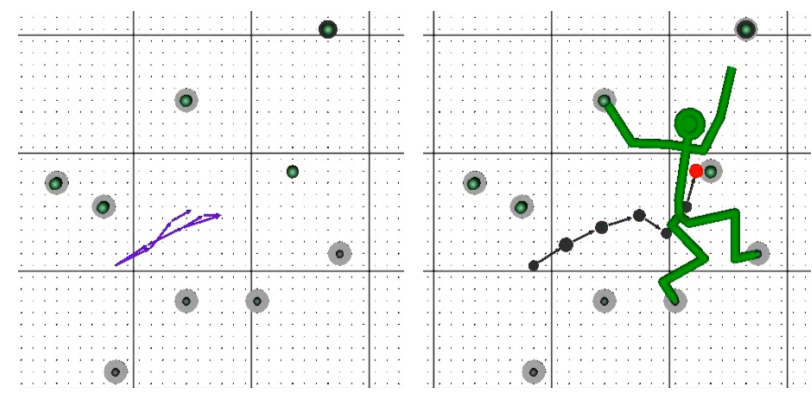

Figure 2: Visualization during (left) and after search (right).

\section{Informal User Study}

We conducted an informal user study with 3 good climbers. Two had a lot of experience in route setting, while one only had created very few routes so far. Each participant was asked to create 3 routes that he would like to climb himself. We explained the software before starting and collected feedback during the whole study.

One user said "one gets funny ideas" for routes using the software and that it's "good for inspiration but you don't get around having ideas of your own". Another participant said it may be difficult for professional route setters to make use of the software as you need to have a computer in the climbing gym and replicate the real wall using the software. But he also stated it's useful for beginners who usually have problems getting the distances between holds right and could be used to simulate a child climber as it is difficult even for experienced route setters to create good routes for children.

All participants commented on the sometimes strange poses and movements. Two participants noted that the climber seems to be too flexible and takes footsteps that are too high. All 3 users also noted that cross moves were frequent and too far (grabbing to the right with the left hand while holding on to the wall with the right, crossing the arms). Human climbers switch hands on a single hold to prevent this, something that we did not implement. They also commented on multiple other missing features:

- Dynamic moves (jumps, dangling feet, using the momentum)

- Using the wall where there are no holds (climbers often just place a foot or a hand against the wall to balance or move up)

- Feedback on why a move fails (real route setters feel their fingers slipping when test climbing)

- Adjustable climber size and skill

- Showing more than one variant to climb the route (quality routes can be climbed in different ways)

Differently shaped holds are of course needed as they form the basis of free climbing. Two participants suggested letting the user rotate the holds as well as adjust how easy they are to grab. Selecting the angle a hold can be grabbed in relation to the wall, is also important according to one user. Some holds can only be pulled at in parallel to the wall and some support a force at a right angle.

The size of the black circles did not register at all with one user and another said if they accurately show the difficulty would depend on the holds used on the real wall. When asked about the spray-paint tool one participant said it could be useful for easy children's routes. Another noted one usually wants something planned (non-random) but that it might be good for passages where you lack an idea.

\section{Conclusion and Future Work}

The climbing route designer allows fast exploration of possible climbing routes by testing variants easily. According to study participants it could be especially useful for beginners or experienced route setters creating climbing routes for children, as well as getting inspiration. One user stated that if the virtual climber climbed better, it could remove the need to test climb every route. According to the users many of the static poses resemble real life climbing poses, but a lot are still unrealistic. The results from our informal user study can be a starting point for implementations using more complex algorithms to generate climber poses and animation.

\section{References}

BRetL, T. 2006. Motion Planning of Multi-Limbed Robots Subject to Equilibrium Constraints: The Free-Climbing Robot Problem. The Intl. Journal of Robotics Research 25, 4, 317-342.

Gill, P. E., Murray, W., ANd Saunders, M. A. 2005. SNOPT: An SQP Algorithm for Large-Scale Constrained Optimization. SIAM Review 47, 1, 99-131.

Kalisiak, M., And VAn de Panne, M. 2001. A grasp-based motion planning algorithm for character animation. The Journal of Visualization and Computer Animation 12, 3, 117-129.

LAnday, J. A. 2009. Design tools for the rest of us. Communications of the ACM 52, 12, 80-80.

Ouchi, H., Nishida, Y., Kim, I., Motomura, Y., And MiZOGUCHI, H. 2010. Detecting and modeling play behavior using sensor-embedded rock-climbing equipment. In IDC 2010.

Phillips, C., AND Bradley, E. 2010. Strange Beta: Chaotic Variations for Indoor Rock Climbing Route Setting. In ICAND 2010, 290-297.

Umetani, N., Kaufman, D. M., Igarashi, T., And GrinSPUN, E. 2011. Sensitive Couture for Interactive Garment Modeling and Editing. In SIGGRAPH 2011, vol. 30. 